\section{Dermatomyositis followed by pemphigus foliaceus: a case report and possible mechanism}

\author{
Ahmad M. Tuffaha, Nabih I. Abdou \\ Center of Rheumatic Disease, Allergy and \\ Immunology, St Lukes Hospital, \\ University of Missouri, School of \\ Medicine, Kansas City, MO, USA
}

Dermatomyositis (DM) and Pemphigus foliaceus (PF) are two autoimmune diseases that are rarely reported in the same patient. DM is an idiopathic inflammatory myopathy with characteristic skin manifestations. Muscle involvement manifests as proximal weakness. Muscle tenderness may occur but is not a regular feature. Classic dermatological findings in DM include Gottron's papules, heliotrope erythema, the shawl sign, and facial erythema. The etiology of DM remains unknown; some studies have reported an association with histocompatability antigens, environmental agents (e.g. virus, drugs) and autoimmunity. ${ }^{1,2}$ Several autoantibodies have been identified, but their routine use in diagnosis has not yet been defined. These autoantibodies have been termed myositis-specific antibodies and include Anti-Mi-2, Anti-Jo-1, antisignal recognition protein and anti-Ku. ${ }^{2}$ Pemphigus foliaceus, which is the least severe form of pemphigus, is characterized by crusted, scaly sores, or fragile blisters. Oral or other mucocutaneous blisters do not occur in this disorder. Circulating antibodies in PF bind to desmoglein-1 protein rather than to the desmoglein-3 protein affected in pemphigus vulgaris (PV). Unlike the blisters seen in PV, the lesions in PF may itch and may easily be confused with eczema or non-specific dermatitis.

We report a case of a 53-year old African American female who presented with DM and later developed PF. Narbutt et al. reported a similar case of a patient who presented with these two conditions. ${ }^{3}$

Our patient was diagnosed with DM in 1989 at the age of 34 years. At that time, the patient complained of proximal muscle weakness along with skin rash over her knuckles. Physical examination showed proximal myopathy along with Gottron's papules. Laboratory investigation showed elevated erythrocyte sedimentation rate (ESR $72 \mathrm{~mm} / \mathrm{h}$ ), and creatine phosphokinase (CPK 1,928 IU/L), while both antinuclear antibodies (ANA) and antiextractable nuclear antigens (anti-ENA) antibodies were negative. Family history of autoimmune diseases was negative. The patient was started on oral prednisone with a maintenance dose of $10 \mathrm{mg}$ daily. Attempts to taper the patient off the steroid resulted in recurrence of the disease, manifested by proximal muscle weakness and elevated CPK and ESR. Methotrexate at a dose of $20 \mathrm{mg} /$ week was added to her regimen to help with the weaning process in 1996, but was discontinued after six months because of minimal benefit. The patient was steroid dependant for 14 years till successful weaning was achieved in 2003 . At that time, the patient continued to have significant muscle weakness. The latter was thought to be steroid induced myopathy rather than active DM since her CPK and ESR were within normal limits. During these years, the patient developed hypertension for which she was started on lisinopril and developed diabetes mellitus requiring insulin treatment. In 2004 , the patient developed an itchy rash on her back that spread to involve the entire body, with sparing of the mucus membranes. Physical examination at that time showed multiple hyperpigmented skin lesions, with skin sloughing over the face, trunk and extremities (Figure 1). Laboratory investigations showed a positive anti-desmoglein-1 with a titer of 236 units (normal level $<20$ units), along with negative anti-desmoglein-3 antibodies. Skin biopsy showed intraepidermal bullous formation with epidermal acute inflammation and acantholysis along with superficial interstitial and perivascular mixed lymphohistiocytic inflammation with polymorphonuclear leukocytes. These findings were consistent with the diagnosis of PF. The patient was started on hydroxychloroquine but with no improvement after six months of therapy. Prednisone was then added and the patient showed significant improvement. Other treatments in her course included mycophenolate mofetil, which the patient did not tolerate due to gastrointestinal problems, and intravenous immunoglobulin at a dose of $1 \mathrm{gm} / \mathrm{kg}$ daily for three days which was beneficial. Unfortunately, the latter was not approved by her insurance. Lisinopril was stopped during that time and valsartan was started instead. Currently the patient is on

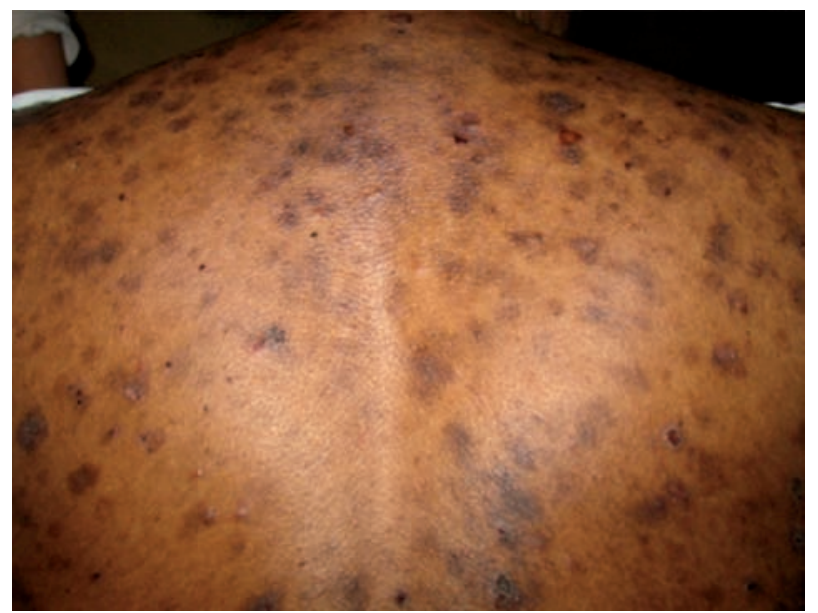

Figure 1. Patient of this report showing her back with multiple hyperpigmented skin lesions of Pemphigus Foliaceus.
Correspondence: Ahmad M. Tuffaha, St Lukes Hospital, 4401 Wornall Rd, Kansas City, M0 64111, USA. E-mail: tuffahaa@umkc.edu

Key words: pemphigus foliaceus, dermatomyositis, plectin, desmoglein.

Contributions: AT is the first author in the writing of this manuscript; NIA is the rheumatologist who diagnosed and followed-up this case, and participated in the writing of this manuscript.

Conflict of interest: the authors reported no potential conflict of interests.

Received for publication: 1 August 2009.

Revision received: 2 October 2009.

Accepted for publication: 5 October 2009 .

This work is licensed under a Creative Commons Attribution 3.0 License (by-nc 3.0).

(C) Copyright A.M. Tuffaha and N.I. Abdou, 2009 Licensee PAGEPress, Italy

Rheumatology Reports 2009; 1:e11

doi:10.4081/rr.2009.e11

prednisone $10 \mathrm{mg}$ every other day with relatively controlled skin manifestations. She continues to have proximal muscle weakness of both upper and lower extremities due to the persistence of steroid induced myopathy.

This patient demonstrates coexistence of PF and DM. This might be a coincidence or a true association. One of the explanations of such an association is that PF might be medication induced in a genetically susceptible person. Our patient was on lisinopril for the treatment of hypertension. Lisinopril contains the thiol group which has been reported to cause druginduced pemphigus. ${ }^{4}$ Our patient was then switched to valsartan. A literature search documented one patient who developed PF after being treated with angiotensin II receptor blocker. ${ }^{4}$ In drug induced PF, most patients go into remission once the offending agent is stopped. In our case, the patient did not 
improve after discontinuation of lisinopril.

Multiple theories have been proposed to discuss the pathogenesis of autoimmune diseases, including the relation to changes in environmental factors. ${ }^{5}$ The latter has strongly been suggested by epidemiological studies of $\mathrm{PF}$ in Brazil, where the incidence of PF declines as the distance increases from the regions where PF is endemic. ${ }^{5}$ Another theory discusses the relation to viral infections which initiate autoreactivity through molecular mimicry. However, these theories focus on how the autoimmune process starts and not on how it progresses. The most acceptable explanation for progression of autoimmune diseases is epitope spreading. ${ }^{5}$ This was reviewed by Chan $e t$ al. who postulated that skin injuries triggered by autoimmune or inflammatory processes could induce autoimmunity to a normally sequestered epitope. B cells will then present new antigens to naïve $\mathrm{T}$ cells, causing activation of the inflammatory process. ${ }^{5}$ In our case, we would like to postulate that DM associated muscle damage results in release of skin and muscle antigens, leading to the formation of autoantibodies that might cross react with epidermal antigens and subsequently to the development of PF. Multiple studies suggest that plectin, a versatile cytoskeletal linker protein, has an essential function in maintaining the integrity of skin and muscle cytoarchitecture. ${ }^{6}$ In DM, muscle damage causes release of plectin. This would result in an autoimmune reaction targeting the plectin in other tissues including the skin. Autoantibodies against skin plectin could lead to the loss of skin integrity causing the release of other desmosome compo- nents including desmoglein with subsequent autoimmune reaction to desmoglein that is seen in PF. This hypothesis is supported by the fact that autoimmunity against plectin was described in paraneoplastic pemphigus. ${ }^{7}$ However, this theory does not explain why in our patient PF developed 15 years after the onset of DM. In our case, DM was diagnosed in a presumably genetically susceptible patient who was prone to develop other autoimmune diseases. The patient was treated with steroid for 14 years before successful weaning was achieved. We would like to speculate that once she was off the steroid, her immune system started forming autoantibodies (antidesmoglein 1). We believe that lisinopril could also have triggered the disease.

The lesson from our case is that two autoimmune diseases can coexist in the same patient because different tissues in the body share common antigens. Moreover, when tapering a patient with an autoimmune disease off steroid or immune suppressive therapies, we should be looking for the possible development of other autoimmune diseases, and not only the relapse of the original disease. Apparently, therapy of multiple autoimmune diseases in the same patient needs further studies. Whether corticosteroids by themselves or a combination of corticosteroids with immunosuppressive drugs are beneficial to treat this association remains controversial. In our case, steroid was an efficient treatment but steroid induced myopathy was disabling to the patient. With the new advancement in modalities in the therapy of autoimmune diseases, it is possible to treat these patients with steroid sparing agents.

\section{References}

1. Callen JP. Dermatological signs of internal disease. 2nd ed, Saunders 1995:13-20.

2. Callen JP, Wortmann RL. Dermatomyositis. Clin Dermatol 2006:24:363-73.

3. Narbutt J, Torzecka JD, Sysa-Jedrzejowska A, Pas HH. Pemphigus foliaceus in an 11year-old boy with dermatomyositis: simple coincidence or familial immunological background? Br J Dermatol 2003; 148:8389.

4. Bae YI, Yun SJ, Lee SC, et al. Pemphigus foliaceus induced by an angiotensin II receptor blocker. Clin Exp Dermatol 2008;33:721-3.

5. Chan LS, Vanderlugt CJ, Hashimoto T, et al. Epitope spreading: lessons from autoimmune skin diseases. J Invest Dermatol 1998;110:103-9.

6. Andrä K, Lassmann H, Bittner R, et al. Targeted inactivation of plectin reveals essential function in maintaining the integrity of skin, muscle, and heart cytoarchitecture. Genes Dev 1997; 11:3143-56.

7. Proby C, Fujii Y, Owaribe K, et al. Human autoantibodies against HD1/plectin in paraneoplastic pemphigus. J Invest Dermatol 1999;112: 153-6. 